\title{
CAMA DE BROILER Y GRANO DE MAIZ ENTERO O MOLIDO EN RACIONES DE ENGORDA INVERNAL DE NOVILLOS.
}

\author{
Claudio Rojas G. ${ }^{1}$
}

Instituto de Investigaciones Agropecuarias. Centro Regional de Investigación Carillanca. Correo

58-D, Temuco. Chile. E-mail:cbrojas@inia.cl

\section{ABSTRACT \\ Broiler litter and ground or whole maize gra- in in winter finishing steers.}

Key words: steers, rate of gain, wheat silage.

The objective of this study was to evaluate the animal response to feeding winter fattening rations based on silages of pure wheat (Triticum aestivum L.) or wheat forage supplemented with broiler litter or a mixture of litter and corn grain. (Zea mays L). Treatments were 1) wheat silage with broiler litter and ground corn offered at feeding time; 2 ) wheat forage mixed with broiler litter at ensiling and ground maize supplemented at feeding time; 3) wheat forage mixed with broiler litter and ground maize at ensiling and 4) wheat forage mixed with broiler litter and whole maize at ensiling time. Daily LW gains (LWG) were 1.296, 1.160, 1.272 and $1.222 \mathrm{~kg} \mathrm{animal}^{-1}(\mathrm{P}>0.05)$; feed consumption was 12.0, 11.6, 12.0 and $12.1 \mathrm{~kg} \mathrm{DM}$ animal $^{-1}$ and feed conversion was 9.3, 10.0, 9.4 and 9.9 $\mathrm{kg}$ DM feed $\mathrm{kg}^{-1}$ LW gain; dressing percent were 54.1, 56.3, 55.3 and $54.5(\mathrm{P}>0.05)$ for treatments $1,2,3$ y 4, respectively. Incorporating broiler litter alone or with whole or milled maize grain in the silage at the ensiling moment did not affect daily LWG and dressing percentage of the steers compared with the silage and supplement mixture delivered at the moment of feeding the steers, and that milled the grain is not necessary.

\section{RESUMEN}

Palabras claves: novillos, incremento de peso, ensilaje de trigo.

El estudio tuvo el objetivo de evaluar la respuesta animal al consumo de raciones de engorda basadas en ensilaje de trigo (Triticum aestivum L.), ensilado solo o ensilajes de trigo con cama de broiler, o con cama de broiler y grano de maíz (Zea mayz L.) entero o molido. Los tratamientos fueron: 1) ensilaje de trigo solo, suplementado con cama de broiler y grano de maíz molido, al momento de la alimentación invernal; 2) ensilaje de trigo mezclado con cama de broiler al ensilar y grano de maíz molido adicionado al momento de alimentar; 3 ) ensilaje de trigo con cama de broiler y grano de maíz molido mezclados al momento de ensilar y 4) ensilaje de trigo mezclado con cama de broiler y grano de maíz entero adicionados al momento de ensilar. Los incrementos diarios de PV fueron de 1,296a; 1,160a; 1,272a y 1,222a kg animal ${ }^{-1}(\mathrm{P}>0,05)$; el consumo de alimentos de $12 ; 11,6 ; 12$ y $12,1 \mathrm{~kg}$ MS ani$\mathrm{mal}^{-1}$; las conversiones de alimentos de 9,$3 ; 10$; 9,4 y 9,9 kg MS alimento consumido $\mathrm{kg}^{-1}$ incremento PV y los rendimientos centesimales de las canales de 54,1a; 56,3a; 55,3a y 54,5a (P > $0,05)$ para los tratamientos $1,2,3$ y 4 , respectivamente. Se concluye que la incorporación al forraje de trigo, al momento de ensilar, con solo cama de broiler o con cama de broiler y grano de maíz, entero o molido, se obtienen similares incrementos de peso y rendimientos de canal a los obtenidos al suplementar el ensilaje con los mismos ingredientes mezclados al momento de alimentar los novillos. Estos resultados no justifican la molienda del grano de maíz. 


\section{INTRODUCCIÓN}

El grano de maíz (Zea mays L.) es un cereal que se utiliza para consumo animal para fabricar concentrados. La demanda de este grano en ganadería deriva de su mayor contenido de energía metabolizable $\left(3,1 \mathrm{Mcal} \mathrm{kg}^{-1}\right)$ y mayor digestibilidad (89\%), respecto a los granos de avena (Avena sativa L.), cebada (Hordeum vulgare L.) y triticale (Triticosecale Wittmack) (Rojas y Catrileo, 2004). Su utilización generalizada en la engorda de novillos es como grano procesado y formando parte de los concentrados.

Al respecto, numerosos estudios señalan que la molienda de los granos de cereales, especialmente cebada y trigo, aumenta la digestibilidad y disponibilidad inmediata de energía y nitrógeno de la ración para la síntesis de proteína microbiana, lo que permitiría buenos incrementos de peso (Pettersson y Martinsson, 1994; Mathison, 1996; Owens et al., 1997). Sin embargo, una molienda muy fina de estos granos puede provocar acidosis subclínicas y clínicas, cuando su proporción en la ración aumenta respecto de los forrajes conservados, siendo las moliendas menos finas las que promueven mayor incremento de peso animal (Owens et al., 1997).

En el caso del maíz, algunos estudios señalan que el consumo de grano entero es, también, una alternativa para bovinos en engorda debido a que la masticación permite dañarlo al punto de no ser necesario su procesamiento físico (Beauchemin et al., 1994; Owens et al., 1997). El proceso de masticación sería mayor en los animales jóvenes y que consumen raciones con adecuados niveles de fibra larga, a diferencias de lo que ocurre en vacas que mastican y rumian menos, necesitando del cereal procesado (Rainey, 2004). Según Vinardell et al. (2005) la actividad masticatoria, ingestiva y de rumia en terneras que consumen grano de maíz entero en la ración, aumenta desde los 10 a los 15 meses para luego mantenerse hasta los 17 meses de edad. Esto es concordante con Gorocica-Buenfil y Loerch (2005) que no obtuvieron diferencias en el consumo, digestibilidad de las raciones ni en las ganancias de peso entre novillos de destete y de engorda alimentados con raciones con grano de maíz entero o molido. En este sentido también Elizalde et al. (2007) determinaron que con raciones de engorda con alto contenido de grano entero de maíz entregadas a novillos de más de un año y $323 \mathrm{~kg}$ de peso se obtenían consumos, incrementos de peso y eficiencias de conversión similares a las obtenidas con raciones similares pero con el grano partido. En forma complementaria a lo señalado Elizalde et al.(2005) determinaron que en vaquillas de año fistuladas de $263 \mathrm{~kg}$ y alimentadas con altos niveles de grano entero de maíz en la ración, sólo cantidades menores se excretaban sin ser dañadas en la rumia, no justificando por tanto su molienda y posibilitando bajar los costos del concentrado a nivel predial.

Por otro lado es usual que los granos de cereales y otros suplementos que se utilizan en raciones de bovinos en engorda se mezclen entre sí junto al forraje conservado, mayoritariamente ensilajes, al momento de la alimentación invernal. Para esta labor se usan desde carros mezcladores, a formas manuales. La alternativa de adicionar los alimentos concentrados al momento de realizar el ensilaje en primavera, permitiría posiblemente disminuir los efluentes y disponer de una ración más equilibrada al abrir el silo, bajando los costos de la mano de obra y maquinaria. Al respecto, la incorporación de cama de broiler al ensilaje de cebada o de praderas al momento de ensilar ha sido señalada por Rojas et al.,(1999) como una práctica que no altera el consumo ni los incrementos de peso vivo de los novillos, en comparación a mezclarlos en el momento de la alimentación invernal. Esto ha sido corroborado por Rojas y Manríquez (2007) quienes no obtuvieron diferencias en consumo ni incrementos de peso al comparar una ración de ensilaje de pradera mezclado con cama de broiler y grano de cebada entero o molido al momento de su elaboración, con otra de ensilaje de pradera solo, suplementada con cama de broiler y grano de cebada al momento de alimentar.

El presente estudio tuvo como objetivo evaluar la respuesta animal al consumo de raciones constituidas por ensilaje de trigo mezclado con cama de broiler y grano de maíz molido o entero, ambos incorporados al momento de ensilar, en comparación a la alternativa de aportar ensilaje de trigo suplementado con cama de broiler y grano de maíz, al momento de alimentar. 


\section{MATERIALES Y METODOS}

El estudio se realizó en el Centro Regional de Investigación Carillanca del Instituto de Investigaciones Agropecuarias (INIA), Temuco ( $38^{\circ} 41^{\text {' }}$ lat. Sur, $72^{\circ} 25^{\prime}$ long Oeste, 200 m.s.n.m.) durante la temporada otoño-invierno de 2001.

Se utilizaron 24 novillos Overos Colorados y Overos Negros de 19 a 20 meses de edad, nacidos en primavera y con $350 \mathrm{~kg}$ de PV inicial promedio. Los tratamientos fueron los siguientes: T1: ensilaje de trigo, cama de broiler y grano de maíz molido, mezclados al momento de la alimentación invernal; T2: ensilaje de trigo mezclado con cama de broiler al momento de ensilar y grano de maíz molido suplementado al momento de la alimentación invernal; T3: ensilaje de trigo con cama de broiler y grano de maíz molido mezclados al ensilar y T4: ensilaje de trigo mezclado con cama de broiler y grano de maíz entero al momento de ensilar.

Los tratamientos se formularon de modo de constituir raciones completas aproximadamente isoproteicas e isoenergéticas, utilizando una relación ensilaje : concentrados de 60 : 40; en la parte concentrada de la ración la proporción de cama de broiler a grano de maíz fue de 65 : 35, BMS

La cama de broiler fue comprada a granel a fines de diciembre y correspondió al desecho del piso de galpones de engorda de pollos broiler (mezcla de viruta y guano de ave), de una temporada, harnereada y seleccionada para evitar la presencia de objetos metálicos, y sometida a homogenización y secado. La cama de broiler que no se incorporó al ensilaje se mantuvo ensacada hasta su utilización invernal.

El grano de maíz, la cama de broiler y el ensilaje se analizaron en el Laboratorio de Bromatología de INIA Remehue, Osorno; la determinación de materia seca (MS), proteína cruda (PC), fibra cruda (FC) y nitrógeno amoniacal (N-NH3), se realizó según los métodos de la AOAC (1970), y la energía metabolizable (EM) se estimó en base a la digestibilidad in vitro (Tilley y Terry, 1963), (Cuadro 1). En este cuadro se observa que el grano de maíz presentó menor contenido de MS, más PC y valores similares en EM y de FC que los señalados en las tablas de composición de alimentos de la UACH (1985) donde los valores son de 86,3\%; 9\%; 3,3 Mcal $\mathrm{kg}^{-1}$ y $2,2 \%$ para los mismos parámetros, respectivamente. La cama de broiler presentó un mayor contenido de MS que lo usual, que es de 77 a $88 \%$, lo que se atribuye a que fue comprada en la zona central del país en el mes de diciembre, cuando las condiciones climáticas son de alta temperatura y baja humedad relativa. También presentó mayor PC y menor EM, FC y N-NH3 que otras partidas de camas utilizadas en INIA Carillanca (Rojas et al., 1999).

El material para producir el ensilaje provino de trigo variedad Dalcahue INIA (Triticum aestivum L.) sembrado el 28 de agosto de 2000 con una dosis de semilla de $200 \mathrm{~kg}$, y una fertilización de $100 \mathrm{~kg}$ de salitre sódico, $320 \mathrm{~kg}$ de superfosfato triple y 80 de cloruro de potasio por hectárea. A la macolla se desparramó en cobertera $300 \mathrm{~kg}$ de urea $\mathrm{ha}^{-1}$. La cosecha de forraje se realizó el 11 de enero del 2001,

Cuadro 1. Composición química de los alimentos usados en la engorda (BMS).

Table 1. Chemical composition of the ingredients used in the fattening rations (DM).

\begin{tabular}{|l|c|c|c|c|c|c|}
\hline Alimentos & $\begin{array}{c}\text { Materia } \\
\text { seca } \\
(\%)\end{array}$ & $\begin{array}{c}\text { Proté́na } \\
\text { cruda } \\
(\%)\end{array}$ & $\begin{array}{c}\text { Energía } \\
\text { metabolizable } \\
\left(\mathbf{M c a l ~ k g}^{-1}\right)\end{array}$ & $\begin{array}{c}\text { Fibra } \\
\text { cruda } \\
(\%)\end{array}$ & $\begin{array}{c}\text { N-NH3 } \\
(\% \text { del N } \\
\text { total })\end{array}$ & $\begin{array}{c}\text { PH } \\
(\mathbf{1}-7)\end{array}$ \\
\hline Ensilaje de trigo & 38,4 & 10,8 & 2,51 & 22,5 & 4,2 & 4 \\
\hline Grano de maíz & 84,6 & 9,31 & 3,32 & 2,2 & n.a. & n.a. \\
\hline Cama de broiler & 92,6 & 24,1 & 1,91 & 21,5 & 14,1 & n.a \\
\hline
\end{tabular}

Determinado en Laboratorio de Bromatología, Instituto de Investigaciones Agropecuarias, CRI Remehue, Osorno. n.a.: No aplicable 
estando el trigo en un estado de desarrollo de grano harinoso suave correspondiente a $84 \mathrm{de}$ la escala de Zadoks et al., (1974). La cosecha de forraje se realizó con una cosechadora de forrajes (New Holland, modelo 16A, USA) y el material se ensiló sobre plástico en cuatro silos tipo parva, realizados en forma simultánea, los que se taparon con una cubierta de plástico y una gruesa capa de tierra.

El primer ensilaje se confeccionó tratando de imitar una ración completa para lo cual se formuló en forma previa un concentrado que tenía una proporción de cama de broiler a grano de maíz molido de 65:35 BMS, el cual se adicionó al forraje de trigo al momento de realizar el ensilado en la proporción de $40 \%$ BMS. El segundo ensilaje se confeccionó igual que el primero pero utilizando grano de maíz entero. Para esto, se realizaron los cálculos considerando el contenido de MS del grano de maíz y de la cama de broiler determinado previamente en laboratorio, así como el contenido de MS del forraje de trigo dos días antes del corte para la confección del ensilaje, (en estufa con aire forzado a $60^{\circ} \mathrm{C}$ durante 2 días).

Al tercer ensilaje se le adicionó cama de broiler en la proporción de $1 \mathrm{~kg}$ por cada 2,32 $\mathrm{kg}$ de forraje de trigo (BMS) para mantener la misma proporción de este producto que se utilizó en los 2 primeros ensilajes. Un cuarto ensilaje fue de ensilaje de trigo sólo, sin concentrados.

Al ensilaje de trigo sólo (cuarto silo) y a aquel con sólo cama de broiler (tercer silo) se les adicionó los suplementos faltantes al momento de alimentar los animales en invierno, en las mismas proporciones utilizadas en los dos primeros ensilajes mezclado todos los ingredientes para aproximarse a una ración completa. Para esto se determinó, aproximadamente cada 20 días, el contenido de MS de los ingredientes concentrados con el objetivo de corregir por humedad las proporciones a usar de cada uno de ellos. Los ingredientes concentrados utilizados (cama de broiler y grano de maíz) fueron de la misma partida incorporada en los ensilajes de los silos primero y segundo.

Todos los tratamientos tuvieron una composición química calculada de 49,7\% MS; $14 \%$ PC; $19,4 \%$ FC y 2,48 EM y se ofrecieron a libre consumo.

Se realizó un período pre-experimental de acostumbramiento de los animales a la estabulación y manejo de alimentación que se inició el 17 de mayo del 2001 y tuvo una duración de 14 días. Además, en este período los animales se desparasitaron contra parásitos hepáticos, gastrointestinales y pulmonares. Ninguno de ellos recibió anabólicos. El período experimental se inició el 1 de junio y tuvo una duración de 81 días. En ambos períodos se utilizó un corral techado por tratamiento, de aproximadamente 60 $\mathrm{m}^{2}$, donde $25 \mathrm{~m}^{2}$ estaban habilitados para cama caliente y $35 \mathrm{~m}^{2}$ con un radier de cemento para el sector de comederos y bebederos. La cama caliente estuvo constituida por paja de trigo, la que se agregó diariamente para mantenerla relativamente seca.

Para determinar incrementos de peso vivo (PV) se realizó un pesaje individual de los animales al inicio del estudio y luego cada 14 días en promedio, sin destare, realizado entre 9 y $10 \mathrm{~h}$ AM. El consumo de alimento se determinó diariamente en forma grupal por diferencia entre la cantidad de alimento que se ofreció dos veces al día, en cantidades totales cercanas al 3\% del PV BMS, y la rechazada que se retiró una vez al día a primera hora de la mañana.

En los animales faenados, previo destare de 20 h, se determinó el rendimiento centesimal de las canales en caliente. Posteriormente, con 24 h en cámara a $2^{\circ} \mathrm{C}$ se estimó la cobertura y color de la grasa.

El análisis estadístico para los incrementos de peso y de rendimiento centesimal se realizó a través de un diseño experimental de bloques completos al azar. Los resultados se analizaron a través de un análisis de covarianza, donde la covariable fue peso inicial. Las diferencias entre las medias corregidas fueron analizadas mediante la prueba t (5\%), (Cochran y Cox, 1974).

\section{RESULTADOS Y DISCUSION}

\section{Consumo de alimentos}

El consumo diario de MS alcanzado por los novillos en los diferentes tratamientos fue de aproximadamente $12 \mathrm{~kg}$ (Cuadro 2), cifra que 
es similar a la obtenida en otros estudios en los que utilizó ensilaje de cereales realizados en similares estados fenológicos y relación de forraje conservado a concentrados. Así, Rojas y Manríquez (2001) obtuvieron consumos de 10,7 y $12,3 \mathrm{~kg}$ en novillos de 19 a 20 meses de edad que consumían raciones de engorda basadas en ensilajes de trigo cosechados en estados fenológicos de grano lechoso harinoso y grano harinoso suave a duro ( 83 y 86 según escala de Zadoks et al., 1974), respectivamente. Rojas et al.,(1999) obtuvieron consumos $12,5 \mathrm{~kg}$ en novillos de igual edad que consumían raciones de engorda con un ensilaje de cebada cosechado en estado de grano harinoso suave equivalente a 85 en la escala Zadoks et al., (1974). Según Oltjen y Bolsen (1980) el consumo de alimentos aumenta en la medida que aumenta el contenido de MS de los ensilajes de trigo, cebada, avena y maíz desde 30 a 41\%. En este rango determinaron aumentos diarios en el consumo de novillos de engorda de $0,08 \mathrm{~kg}$ por cada aumento porcentual de MS de los ensilajes, concluyendo que el consumo se afecta mas por la composición química que por la especie ensilada.

Entre tratamientos se observa un menor con- sumo diario de los novillos en el tratamiento T2 (trigo y cama de broiler incorporada al ensilar), respecto a los otros tratamientos. La diferencia en consumo entre los tratamientos 1 y 2 , que aunque no se hizo análisis estadístico, no coincide con la similitud obtenida en otros estudios que compararon un ensilaje de cebada y cama de broiler y un ensilaje de praderas con cama de broiler realizados en primavera versus estos ingredientes adicionados a los respectivos ensilajes puros al momento de alimentar el ganado en otoño e invierno (Rojas et al., 1999; Rojas y Manríquez, 2007).

La similitud en el consumo diario de las raciones con grano de maíz molido o entero (T3 y T4) estaría señalando que la oferta de maíz entero en raciones de engorda, bajo las condiciones de este estudio, sería una alternativa para bajar costos por concepto de molienda en las raciones de engorda. Estos resultados son concordantes con los obtenidos por Gorocica-Buenfil y Loerch, (2005) y Elizalde et al., (2007) que no obtuvieron diferencias significativas en consumo de raciones constituidas con grano entero de maíz respecto de las con grano molido y partido. Esto se explicaría como consecuencia de la

Cuadro 2.Consumo diario de alimentos al natural y base MS por animal (kg).

Table 2. Daily food intake per animal (kg, as fed and DM basis).

\begin{tabular}{|l|c|c|c|c|}
\hline Consumo al natural & T1 & T2 & T3 & T4 \\
\hline Ensilaje de trigo & 18,78 & - & - & - \\
\hline Cama de broiler & 3,36 & - & - & - \\
\hline Grano de maíz molido & 1,96 & 1,90 & - & - \\
\hline Ensilaje de trigo y cama broiler & - & 21,46 & - & \\
\hline $\begin{array}{l}\text { Ensilaje de trigo, cama broiler y grano de maíz } \\
\text { molido }\end{array}$ & & & 24,19 & \\
\hline $\begin{array}{l}\text { Ensilaje de trigo cama broiler y grano de maíz } \\
\text { entero }\end{array}$ & & & & 24,35 \\
\hline Total base fresca (kg/animal) & 24,12 & 23,36 & 24,19 & 24,35 \\
\hline Consumo MS (kg/animal) & 12,0 & 11,6 & 12,0 & 12,1 \\
\hline
\end{tabular}

T1: ensilaje de trigo, cama de broiler y grano de maíz molido, mezclados al momento de la alimentación invernal; T2: ensilaje adicionado con cama de broiler al momento de ensilar y grano de maíz molido adicionado al momento de la alimentación invernal; T3: ensilaje adicionado con cama de broiler y grano de maíz molido al momento de ensilar; y T4: ensilaje adicionado con cama de broiler y grano de maíz entero al momento de ensilar. 
masticación que permite dañar el grano de maíz entero siendo este proceso mas marcado en animales jóvenes que consumen raciones con niveles de forrajes de $60 \%$ o mas (Beauchemin et al., 1994; Rainey 2004; Vinardell et al., 2005), tal como ocurrió en el presente estudio. En este sentido Elizalde et al., (2005) determinaron en vaquillas de año, alimentadas con raciones que contenían un $79 \%$ de grano de maíz entero, que sólo un 5,8\% se excretaba en las fecas sin ser dañado por la rumia, lo cual estaría señalando que la masticación permite dañarlo al punto de no ser necesario su procesamiento físico.

El consumo de MS como porcentaje del PV promedio de los animales fue de 3; 2,9; 3 y $3 \%$ para los tratamientos 1 al 4 , respectivamente; estas cifras son superiores a la señaladas en tablas de alimentación (ARC, 1980) en función de la proporción de concentrado a forraje conservado usado en la ración y del peso y tipo de animal empleado en la engorda invernal. Sin embargo es similar al obtenido con novillos alimentados con ensilaje de trigo en el estado fenológico de grano harinoso suave a duro, correspondiente a 86 de la escala de Zadocks et al. (1974), cuando la proporción de ensilaje a concentrado fue similar a la usada en este estudio (Rojas y Manríquez, 2001).

\section{Incrementos diario y conversión de alimento}

El incremento diario de peso vivo en los novillos alimentados con el ensilaje de trigo y cama de broiler (T2), tendió a ser menor respecto al de los otros tratamientos, aunque no significativo $(\mathrm{P}>0,05)$. Esta tendencia sería consecuente con el menor consumo de MS observado y la tendencia a una menor eficiencia de conversión (Cuadro3). Sin embargo, destaca la similitud en los incrementos de PV $(\mathrm{P}>0,05)$ de los tratamientos de ración completa que consideraron grano de maíz molido y entero (T3 y T4) y que concuerda con lo observado por numerosos estudios que han comparado el efecto del procesamiento del grano de maíz en la engorda de novillos (Owens et al., 1997 ; Maresca et al., 2002; Dillon y Elizalde, 2005; Elizalde et al., 2007).

La similitud de los incrementos de peso enre raciones con grano entero y molido se explicaría según Owens et al. (1997) por el proceso de masticación que realizan los bovinos, que lo daña en diferente grado, promoviendo un mayor pasaje de partículas de almidón sin fermentar hacia el tracto digestivo posterior, con la consecuente mejor eficiencia de utilización de la energía (almidón), lo que compensaría el mayor gasto de energía derivada de la mayor actividad de rumia al consumir granos enteros .

Las conversiones del alimento fueron de 9,3; 10; 9,4; y 9,9 kg de alimento BMS por cada kilogramo de incremento de PV, para los tratamientos 1, 2, 3 y 4, respectivamente. Las conversiones del alimento alcanzadas se encuentran dentro de los niveles normales, en términos absolutos, para raciones de engorda en base a cereales y animales de razas de carne y de doble propósito (Rojas et al., 1999; Rojas y Catrileo, 2000; Rojas y Manríquez, 2001).

\section{Características de las canales}

Todas las canales mostraron adecuadas cubiertas de grasa, clasificadas en grado 1 de acuerdo a la Norma Chilena de Tipificación (INN, 1993) y de un color amarillo claro. El rendimiento centesimal en caliente de las canales no mostró diferencias estadísticas entre tratamientos $(\mathrm{P}>$ $0,05)$, siendo sus valores promedios de $55,1 \%$ y estimados como normales, al compararlos con estudios de novillos engordados con raciones y genotipo similares (Rojas y Manríquez, 2007) y menores cuando las raciones fueron similares, pero empleando un genotipo menos precoz como el Normando, donde el rendimiento promedio fue de 58,5\% (Rojas y Manríquez, 2001) (Cuadro 3).

\section{CONCLUSIONES}

La incorporación al forraje de trigo de cama de broiler sola o con grano de maíz entero o molido al momento de realizar el ensilaje, en la forma de ración completa, no afectó los incrementos de peso vivo ni los rendimientos centesimales de los novillos, en comparación a suplementar el ensilaje de trigo con los mismos suplementos al momento de alimentar los animales

La similitud de los incrementos de peso y rendimientos centesimales obtenidos en novillos alimentados con raciones que contenían maíz 
Cuadro 3.Respuesta productiva de novillos estabulados con raciones de engorda en base a grano de maíz entero o molido y cama de broiler

Table 3. Productive response of penned steers with fattening rations based on whole or milled corn

\begin{tabular}{|c|c|c|c|c|}
\hline & T1 & $\mathbf{T 2}$ & T3 & T4 \\
\hline Período experimental, días & 81 & 81 & 81 & 81 \\
\hline Peso inicial, $\mathrm{kg}$ animal ${ }^{-1}$ & 348 & 347 & 353 & 352 \\
\hline Peso final, $\mathrm{kg}_{\text {animal }}{ }^{-1}$ & 453 & 441 & 456 & 451 \\
\hline Incremento diario, $\mathrm{kg}$ animal $^{-1}$ & $1,296 a$ & $1,160 \mathrm{a}$ & $1,272 \mathbf{a}$ & $1,222 \mathrm{a}$ \\
\hline Consumo diario, $\mathrm{kg} \mathrm{MS}$ animal ${ }^{-1}$ & 12,0 & 11,6 & 12,0 & 12,1 \\
\hline Conversión alimentos, $\mathrm{kg} \mathrm{kg}^{-1}$ & 9,3 & 10 & 9,4 & 9,9 \\
\hline Rendimiento centesimal de canales, \% & $54,1 \mathrm{a}$ & $56,3 a$ & $55,3 a$ & $54,5 \mathrm{a}$ \\
\hline
\end{tabular}

Letras diferentes en sentido horizontal señalan diferencias estadísticas significativas, según Duncan $(\mathrm{P}<0,05)$

T1: ensilaje, cama de broiler y grano de maíz molido, mezclados al momento de la alimentación invernal; T2: ensilaje adicionado con cama de broiler al momento de ensilar y grano de maíz molido adicionado al momento de la alimentación invernal; T3: ensilaje adicionado con cama de broiler y grano de maíz molido al momento de ensilar; y T4: ensilaje adicionado con cama de broiler y grano de maíz entero al momento de ensilar.

entero o molido, confirman que no se justifica la molienda de este grano para alimentar novillos.

\section{BIBLIOGRAFIA}

AOAC. 1970. Official methods. William Horwist (ed). Association of Official Agricultural Chemist, 11th ed. Washington D.C., USA. $1015 \mathrm{p}$

ARC. 1980. The nutrient requirements of ruminants livestock. Agricultural Research Council (ARC), Commonwealth Agricultural Bureaux, Farnham Royal, England. $351 \mathrm{p}$

BEAUCHEMIN, K.A.; ALLISTER, M.C.; DONG, Y.; B.I. FARR, B.I.; CHENG, K.J. 1994. Effects of mastication on digestion of whole cereal grains by cattle. J. Anim. Sci. 72:236-246

COCHRAN, W.; COX. G 1974. Diseños experimentales. Mexico, Editorial Trillas. 661p.

INN. 1993. Canales de bovinos. Definiciones y tipificación. Norma Chilena Oficial NCh. 1306, Of. 93. Instituto Nacional de Normalización (INN), Santiago, Chile.

DILLON, A.; ELIZALDE. J.C. 2005. Efecto del genotipo y procesamiento del grano de maíz sobre la producción de carne de novillos alimentados a corral. Revista Argentina de Producción Animal 25 (suppl. 1): 27-28

ELIZALDE, J.C.; DILLON, A.; ZANIBONI, C.M. 2007. Novillos en terminación alimentados a corral con grano de maíz entero y/o partido. Revista
Argentina de Producción Animal 27 (suppl. 1): 44-45

ELIZALDE, J.C., MENCHÓN, P.A.; PARRA, V.F.; MONTIEL, M.D.; EYERARBIDE. G. 2005. Degradación ruminal in situ de la materia seca de dos genotipos de maíz ofrecidos entero o molido, en dietas con alta inclusión de grano, a vacunos. Revista Argentina de Producción Animal 25 (suppl. 1):34-35

GOROCICA-BUENFIL，M.A.; LOERCH, S.C. 2005. Effect of cattle age, forage level, and corn processing on diet digestibility and feedlot performance. J. Anim. Sci. 83:705-714.

MARESCA, S.; SANTINI, F.J.; PAVAN, E. 2002. Comportamiento productivo de terneras alimentadas a corral con grano de maíz entero y partido. Revista Argentina de Producción Animal 22 (supl. 1): $163-168$.

MATHISON, G.W. 1996. Effects of processing on the utilization of grain by cattle. Anim. Feed Sci. Technol. 58:113-125.

OLTJEN, J.W.; BOLSEN. 1980. Wheat, barley, oat and corn silages for growing steers. J.Anim Sci. 51:958-965.

OWENS, F.N.; SECRIST, D.S.; HILL, W.J.; GILL, D.R.1997. The effect of grain source and grain processing on performance of feedlot cattle: a review. J. Anim. Sci. 75:868-879.

PETTERSSON, T.; MARTINSSON. K.A. 1994. Digestibility of whole or rolled ensiled barley grain fed to heifers or lactating cows. Swedish J. Agric. Res. 24:109-113. 
RAINEY, B.M. 2004. Effect of beef cattle age, gender and barley grain processing method on rate and efficiency of gain and nutrient digestibility. M.S. thesis. Bozeman, Montana State University. 86p

ROJAS, C.; CATRILEO, A. 2000. Evaluación de ensilaje de cebada en tres estados de corte en la engorda invernal de novillos. Agricultura Técnica (Chile) 60:370-378.

ROJAS, C.; CATRILEO. A. 2004. Alimentación del ganado. In Rojas, C. (ed.) Manual de producción de bovinos de carne para la VIII, IX y X Regiones. Instituto de Investigaciones Agropecuarias, Centro Regional de Investigación Carillanca, Fundación para la Innovación Agraria, Temuco, Chile. pp 85104

ROJAS, C.; MANRÍQUEZ, M. 2001. Comparación de ensilaje de trigo y de maíz en la engorda invernal de novillos. Agricultura Técnica (Chile) 61: 444-451.

ROJAS, C.; MANRÍQUEZ, M. 2007. Cama de broiler y grano de cebada entero o molido en raciones de engorda invernal de novillos. Agricultura Técnica (Chile) 67: 94-99.
ROJAS, C.; CATRILEO, A.; ZANETTI, M.A. 1999. Uso de cama de broiler como suplemento proteico en raciones de ensilajes de cebada y praderas en la engorda invernal de novillos. XXIV Reunión Anual Sociedad Chilena de Producción Animal (SOCHIPA). Temuco, Chile. 27-29 de octubre. pp.122-123.

TILLEY, J.M.; TERRY, R.A. 1963. A two stage technique for the in vitro digestion of forage crops. J. Br. Grassl. Soc. 18:104-111.

UACH. 1985. Composición de alimentos para el ganado en la zona sur. Universidad Austral de Chile. Facultad de Ciencias Agrarias. Instituto de Producción Animal. Valdivia, Chile. 46 p.

VINARDELL, M.; SANTINI, F.J.; DEPETRIS, G.; FAVERIN, C.; MARESCA, S.; ELIZALDE, J.C. 2005. Influencia de la edad y de la suplementación con grano de maíz entero o partido sobre la actividad masticatoria en bovinos. Revista Argentina de Producción Animal 25 (suppl. 1): 36-38.

ZADOKS, J.; CHANG, T.; KONZAK, C. 1974. A decimal code for the growth stage of cereals. Weed 